\title{
On the use of Unmanned Aerial Vehicles for Antenna and Coverage Diagnostics in Mobile Networks
}

\author{
María García Fernández, Student Member, IEEE, Yuri Álvarez López, Senior Member, IEEE, and \\ Fernando Las-Heras Andrés, Senior Member, IEEE
}

\begin{abstract}
The capability of in-situ antenna measurement and diagnostics using a compact low-cost Unmanned Aerial System is presented. An onboard power sensor collects amplitude-only measurements, which are processed by means of an iterative phase retrieval technique. This technique allows recovering an equivalent currents distribution which characterizes the Antenna Under Test (AUT). These currents can be used for antenna diagnostics as well as to evaluate the field radiated by the AUT. In the field of mobile networks, the proposed system is of interest for rapid assessment of the antennas operational conditions providing radioelectric coverage within the mobile network cells and allowing the evaluation of antenna failures, such as incorrect tilt or beamforming/feeding network malfunction. Proof-of-concept of the system is conducted for testing a commercial Base Transceiver Station (BTS) antenna under normal and malfunctioning operation conditions, discussing the extension of the system to allow measurements at millimeter-wave frequency bands.
\end{abstract}

Index Terms-Radio coverage, antenna measurement, antenna diagnostics, Unmanned Aerial Vehicles (UAVs), phaseless measurements, Near-Field to Far-Field transformation (NF-FF), Sources Reconstruction Method (SRM).

\section{INTRODUCTION}

$\mathrm{T}$ HE development of $5 \mathrm{G}$ standards have put together efforts from different institutions in order to define the radio access and core network architectures capable of facing the challenge of connecting billions of heterogeneous end-user devices, while keeping backward compatibility with existing wireless networks [1]. 5G radio access network seems to rely mainly on new multiple access schemes, based on massive MIMO (Multiple Input Multiple Output) and beamforming technologies [2], that will require the replacement or upgrading of existing Base Transceiver Station (BTS) antennas.

Concerning the deployment of $5 \mathrm{G}$ technologies and networks, several working frequency bands have been proposed, focusing primarily in the $24-28 \mathrm{GHz}$ frequency band [3]. Traditional lower frequency bands such as $700 \mathrm{MHz}$,

This work has been supported by the "Ministerio de Economía, Industria y Competitividad. Gobierno de España" of Spain/FEDER under project TEC2014-54005-P (MIRIIEM), TEC2014-55290-JIN (PORTEMVISION), and grant FPU15/06341; and by the Principado de Asturias, through the Plan de Ciencia, Tecnología e lnnovación 2013-2017 under project GRUPIN14-114.
2.6 GHz, and 3.4-3.8 GHz are also of interest for $5 \mathrm{G}$ deployment thanks to the lower free space propagation losses with respect to mm-wave frequency bands.

Network densification is expected to be another strategy for a successful deployment of $5 \mathrm{G}$ networks [4]. This strategy will result in an increase of the number of cells, reducing propagation path losses especially for mm-wave frequency bands [5].

Taking into account the aforementioned challenges and requirements, one of the fields of interest for the deployment of $5 \mathrm{G}$ networks is the improvement of the methods for radioelectric coverage prediction and calculation, aiming to optimize the placement of the antennas. In this sense, techniques based on antenna characterization using an equivalent currents model [6],[7] or modal expansion [8] provide accurate estimation of the radiated field, not only for coverage evaluation, but also for exposure to radiated fields compliance assessment.

Besides, the increase on the number of coverage cells and thus, the number of antennas, will require the development of new techniques for the assessment of parameters such as power level and radioelectric coverage, as well as for quick, low-cost detection of anomalies in the radiofrequency front-end. Due to the large scale number of cells in 5G networks, the development of such techniques would result in operational and maintenance cost savings.

Thanks to the development of Unmanned Aerial Vehicles (UAVs) in terms of cost reduction and improvement of technical capabilities, they have been successfully introduced in different areas related to communications networks, such as network coverage extension [9],[10] and in-situ antenna measurement and testing [11],[12],[13]. The latter allows the evaluation of the antenna radiation pattern in realistic conditions, analyzing the impact of the surroundings (e.g. multipath contributions in ground, buildings, etc.). The price to pay is the loss of accuracy with respect to anechoic chamber facilities.

In-situ UAV-based antenna measurement in large open areas can be conducted directly in the far field (FF) region of the antenna, which can be hundreds of meters away in the case of

M. García, Y. Álvarez, and F. Las-Heras are with the Área de Teoría de la Señal y Comunicaciones, Universidad de Oviedo. Edificio Polivalente, Módulo 8, Campus Universitario de Gijón.E-33203, Gijón, Spain Email: mariagarcia@tsc.uniovi.es, alvarezyuri@uniovi.es, flasheras@uniovi.es. 
electrically large antennas or at lower operation frequencies (typically less than $1 \mathrm{GHz}$ ). In the scope of $5 \mathrm{G}$ networks it would be the case of macro cell antennas, whose coverage area can range up to $2-3 \mathrm{~km}$. But for micro and nano cell antennas, testing will be likely to occur in the near field (NF) region of the antenna, especially those working at millimeter-wave frequency bands.

The main advantages of FF over NF measurements are:

i) Maximum separation between measurement points is proportional to the distance from the Antenna-Under-Test (AUT). Thus, positioning and data geo-referring errors has more impact in NF measurements.

ii) Radiation pattern has to be measured in the AUT FF region. Otherwise, Near-Field to Far-Field (NF-FF) transformation is required.

However, NF measurements are of interest under the following circumstances:

i) Reducing issues regarding flight autonomy (and thus the number of acquisition points), as FF measurements result in longer flight paths.

ii) Overcoming flight restrictions that may limit the scan zone (e.g. flying over crowded areas, restricted zones, beyond visual line of sight) and therefore, the practical application of FF systems.

iii) Antenna diagnostics cannot be conducted using amplitude-only information collected in the FF region, as explained in [7].

A UAV-based antenna measurement system capable of operating not only in the FF region, but also in the NF of the AUT combines the advantages of AUT measurement in these regions.

Furthermore, antenna diagnostics techniques have been proved to be a quite efficient way of detecting malfunctioning elements in antenna arrays [6],[7],[14]. As mentioned before, it is expected that $5 \mathrm{G}$ networks will implement MIMO and beamforming techniques, which require the use of antenna arrays. Once these antennas are in their final placement within the mobile network, in case of antenna or feeding/beamforming network failure, it is not possible to assess the impact on the cell coverage. Although in most cases the solution is just the replacement of the antenna by the mobile network operator, in some scenarios this is not straightforward. Thus, the possibility of having a-priori diagnostics of the problem would allow the company to decide whether the antenna has to be replaced, it can be fixed in-situ, or the degraded operational conditions are sufficient to maintain the service.

A UAV-based system for antenna diagnostics and characterization (Unmanned Aerial System for Antenna Measurement, UASAM) capable of performing NF antenna measurement and diagnostics has been presented and described in [11]. In this contribution the goals and novelties are: i) to prove the successful application of UASAM for rapid assessment of mobile networks commercial antennas, not only for diagnostics, but also for evaluating the impact of the failure in the antenna coverage; ii) to validate the capability of UASAM to conduct measurements at millimeter-wave frequency bands.

\section{Proof of COncept: In-Situ Antenna MeAsurement SYSTEM}

The proof-of-concept for in-situ measurement and diagnostics of mobile networks antennas using unmanned aerial vehicles will be based on the hardware and post-processing techniques of the system developed in [11] (UASAM), conceived for general-purpose antenna measurement. Fig. 1 provides an overview of UASAM subsystems and postprocessing algorithms, which will be briefly described in this section.

Onboard the UAV there is a monopole probe antenna connected to a power detector, and a Real Time Kinematic (RTK) beacon acting as a rover station for the RTK system.

A second RTK beacon acting as a base station system is placed at a fixed position. The RTK system, together with a laser rangefinder for accurate positioning in height, are in charge of providing centimeter-level in-flight navigation and data geo-referring accuracy.

The data sent by the UAV (geo-referred radiated field measurements and flight path positions coordinates) are postprocessed in a ground station (a laptop).

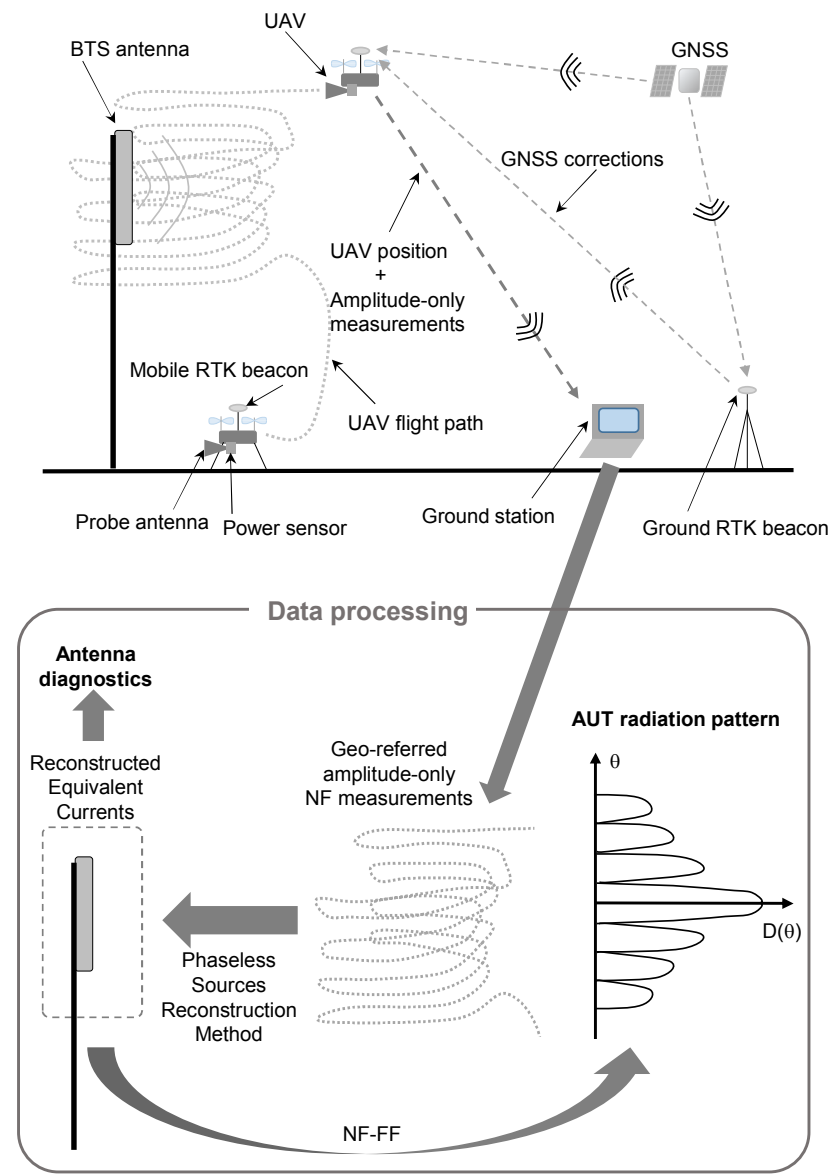

Fig. 1. Block diagram of the Unmanned Aerial System for Antenna Measurement (UASAM).

UASAM makes use of pre-defined flight paths around the AUT, created using waypoints, so the flight path can be repeated if needed. This is of interest in case of in-situ mobile 
network antenna measurements, as the network operator can store the coordinates of these antennas, thus making the procedure of antenna inspection easier.

Communications system between UAV, RTK beacons, and the ground station of the implemented prototype is based on a Wireless Local Area Network (WLAN) operating in the 2.4-2.5 $\mathrm{GHz}$ and/or 5.7-5.8 GHz frequency bands, whereas a $433 \mathrm{MHz}$ radio transmitter and receiver is used for UAV flight control. The use of these frequency bands avoids overlapping with those used by mobile networks, especially for future $5 \mathrm{G}$ communications at millimeter-wave frequencies.

Thanks to the development of both Global Navigation Satellite Systems (GNSS) and RTK technology, multiconstellation and dual-band RTK systems are capable of providing positioning and geo-referring accuracy up to $5 \mathrm{~mm}$, enabling measurements up to $30 \mathrm{GHz}$ (to ensure that georeferring uncertainties are not greater than half wavelength). There are other possible solutions for mm-level accuracy data geo-referring such as laser trackers, as already proposed in [13] for crane-assisted antenna measurement at mm-wave frequency bands. For this proof-of-concept, UASAM makes use of a lowcost RTK system providing $1-2 \mathrm{~cm}$ geo-referring and positioning uncertainty.

Radiated field measurements conducted with power sensors do not allow direct measurement of the phase of the field. Thus, phase retrieval methods are applied to reconstruct the phase from amplitude-only measurements of the radiated field collected on two or more acquisition surfaces around the AUT. UASAM makes use of the phaseless Sources Reconstruction Method (SRM), an iterative phase retrieval technique where an equivalent currents distribution is recovered on a surface enclosing the AUT [7]. These phase retrieval techniques have been successfully tested at frequencies as high as $140 \mathrm{GHz}$, also in the field of antenna measurement for $5 \mathrm{G}$ communications [15].

In addition to this, it must be pointed out that the SRM is capable of handling arbitrary-geometry acquisition domains, thus overcoming the restriction of requiring accurate UAV navigation. As proved in [11], UAV flight path deviation with respect to the pre-defined one has negligible impact in the measurements, as long as they are accurately geo-referred. This feature is of special interest as operational conditions (e.g. wind, propellers vibrations) restrict UAV capability to follow the pre-defined path with cm-level accuracy, which would have prevented the use of UAV for antenna measurement at mmwave frequency bands.

The reconstructed equivalent currents provide an accurate electromagnetic model of the AUT, which can be used for evaluating the radiated field at any point of the space. Immediate applications are radiation pattern calculation and prediction of the radioelectric coverage. Besides, these equivalent currents can be used to determine the presence of malfunctioning elements in the case of antenna arrays. Thus, if an element of the antenna array is not fed, the equivalent currents distribution over this element will exhibit low or zero amplitude.

A monopole antenna is selected as a probe for the proof-of- concept presented in this contribution. The reason of this choice is due to the low directivity and the rotation symmetry of its pattern, which minimizes AUT measurement distortion due to the probe antenna pattern. The selected monopole antenna operates at 900, 1800, and $2350 \mathrm{MHz}$ (in agreement with the working frequency bands of UASAM), having 3\% bandwidth. Similar low-cost monopole or monopole-like antennas are available and ready-to-mount on the UAV for antenna measurement at higher frequency bands.

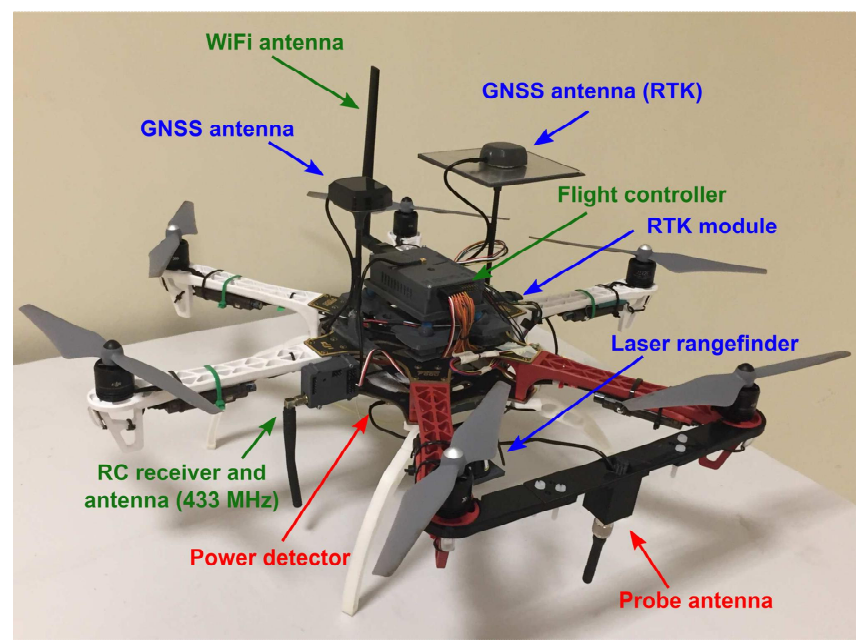

Fig. 2. UAV with the RTK and the probe monopole antenna onboard. Labels in red color: antenna measurement subsystem. Labels in blue: UAV positioning and geo-referring subsystem. Labels in green: communications and UAV controller subsystem.

Technical details and specifications of the rest of devices onboard the UAV are given in [11]. A picture of the UAV with the RTK and the probe printed monopole antenna onboard is shown in Fig. 2.

\section{Mobile Network AnTEnNa Characterization}

Practical assessment of UASAM capabilities for mobile network antenna measurement and diagnostics is described in this section. For this purpose, a $1.3 \mathrm{~m}$ length commercial BTS antenna for GSM and UMTS mobile networks, working at 1800, 2100, and $2300 \mathrm{MHz}$ has been selected (Fig. 3(a) and Fig. 4(a)) to ensure that the positioning and geo-referring error is less than half wavelength at the working frequency. Measurements have been conducted at the airfield located at the Technical School of Engineering of Gijón (coordinates 43.521698, -5.623983).

The BTS antenna has been mounted on top of a $3.5 \mathrm{~m}$ height mast. RTK base station (ground beacon) is deployed 20-25 m away from the base of the mast. Fig. 3(a) shows the placement of the BTS antenna with the UAV in operation, the RTK base station, and the ground station (laptop). For this setup, the WLAN connecting ground RTK beacon, UAV, and the ground station is set to $5.8 \mathrm{GHz}$ to minimize the risk of interferences.

Aiming to simulate antenna malfunctioning, e.g. a failure in one of the elements of the BTS antenna, a sheet of aluminum foil has been wrapped around the lower section of the BTS antenna radome (depicted in Fig. 4(e)). The placement of the 
aluminum foil has been selected so that it does not impact BTS antenna return losses with respect to normal operation conditions.

BTS antenna is fed with a RF signal generator, as shown in Fig. 3(a), tuned at $2350 \mathrm{MHz}$, in order to test UASAM measurement capabilities at the highest working frequency band of the selected BTS antenna.
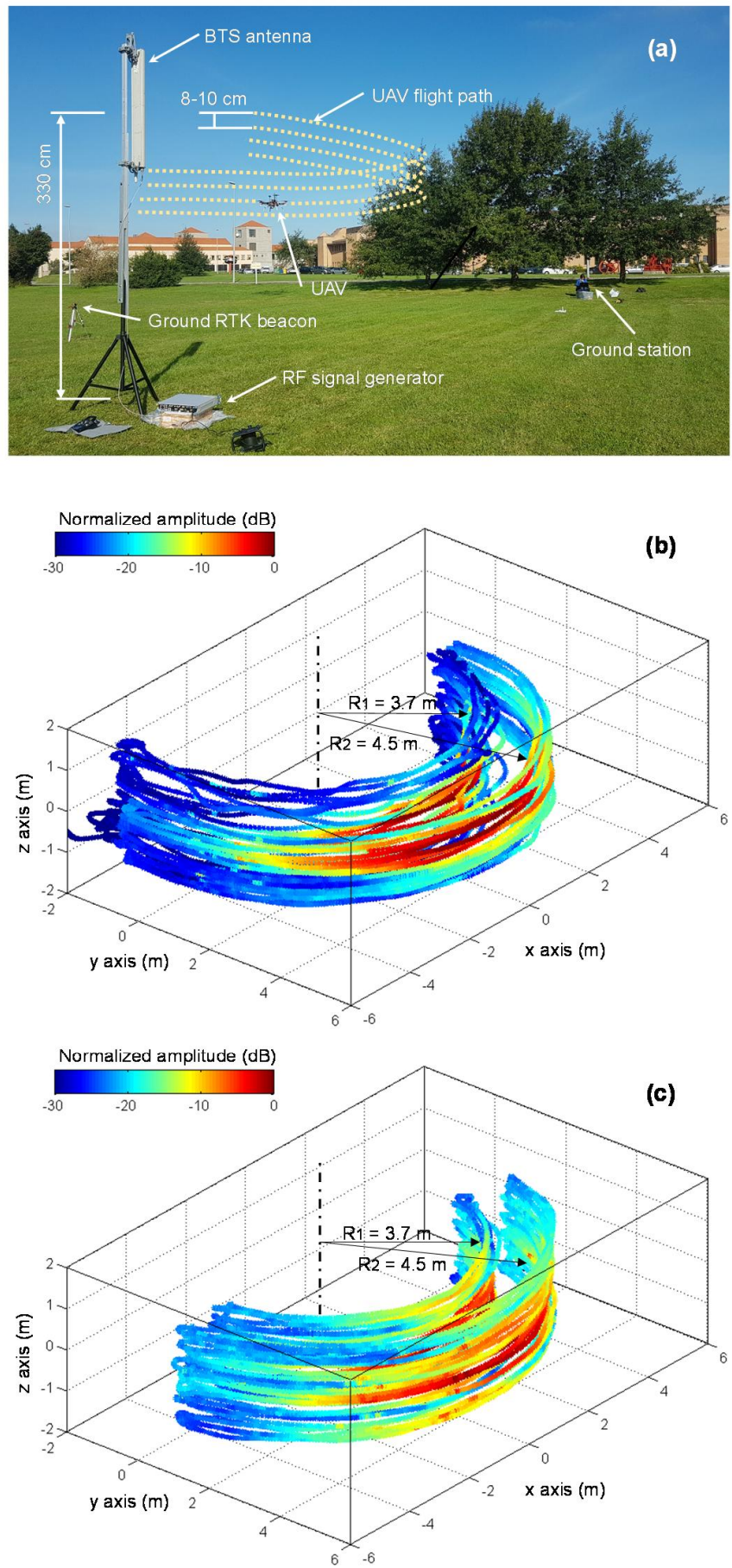

Fig. 3. (a) In-situ measurement of the BTS antenna with the UASAM. (b) BTS antenna under normal operation conditions. (c) Malfunctioning BTS antenna.

In order to set up the flight path around the BTS for radiated field measurement, first the coordinates of a reference point in the base of the BTS antenna are taken [11]. Due to the fact that the expected BTS antenna radiation pattern will cover less than $180^{\circ}$ in the horizontal plane, with little back radiation, the UAV flight path can be fitted to a $180^{\circ}$-arc cylindrical surface. This cylindrical grid ranges from $2 \mathrm{~m}$ to $4.5 \mathrm{~m}$ in height. As mentioned before, iterative phase retrieval techniques require the field to be measured in, at least, two acquisition surfaces around the AUT [7], so two arc cylindrical surfaces with radius $\mathrm{R}_{1}=3.7 \mathrm{~m}$ and $\mathrm{R}_{2}=4.5 \mathrm{~m}$ have been considered. Taking into account the electric size of the BTS antenna, these measurement surfaces are well within the NF region of the BTS (which starts at around $25 \mathrm{~m}$ from the antenna).

Once the flight paths have been defined and uploaded into the UAV controller, the UAV operator takes it off. Then, UAV flight mode is changed from GPS-assisted manual flight mode to autonomous mode so the UAV can follow the pre-defined flight path while taking radiated field measurements. Flight time required to complete the pre-defined path ranges from 12 to 14 minutes for each $180^{\circ}$-arc cylindrical surface. Around 7000 to 8000 geo-referred measurements are collected. A video showing the UAV in operation can be watched at https://youtu.be/4CSGMyl 3OQ.

Geo-referred BTS antenna NF measurements (depicted in Fig. 3(b)) are then processed with the iterative phase retrieval technique based on the SRM to recover an equivalent currents distribution on the BTS antenna aperture plane [7]. That implies minimizing a non-linear cost function that has around 7500 equations and 600 unknowns. With the development of computational capabilities, it can be done with a conventional laptop, requiring not more than 2 minutes calculation. Results for the BTS antenna in normal operation conditions are shown in Fig. 4(b), noticing that the equivalent currents exhibit uniform amplitude along the entire length of the BTS antenna.

In order to provide a reference framework to assess the accuracy of antenna diagnostics from in-situ measurements, the selected BTS antenna has been measured at the spherical range in anechoic chamber of the University of Oviedo. Anechoic chamber measurements have amplitude and phase information, so either amplitude and phase or phaseless NF-NF backpropagation techniques based on the SRM [7] can be applied to obtain an equivalent currents model of the BTS antenna.

Fig. 4(c) shows the reconstructed equivalent currents from measurements in anechoic chamber using amplitude and phase information, whereas Fig. 4(d) corresponds to the reconstructed equivalent currents considering only the amplitude of anechoic chamber measurements.

The same methodology has been applied to the measurements corresponding to the malfunctioning BTS antenna depicted in Fig. 3(c). Results from in-situ measurements are depicted in Fig. 4(f). Fig. 4(g) and Fig. 4(h) correspond to the reconstructed equivalent currents from measurements in anechoic chamber considering amplitude and phase, and amplitude-only information, respectively. In all the three compared cases, equivalent currents vanish in the area where the aluminum foil is placed. 


\section{RADIOELECTRIC COVERAGE ANALYSIS}

The equivalent currents reconstructed from AUT radiated field measurements can be used not only for antenna diagnostics [7],[6], but also for evaluating the field radiated by the AUT at any point, allowing far field pattern calculation, and radioelectric coverage analysis. The latter feature is of special interest to evaluate the impact of commercial BTS antenna failure or misalignment.

Reference BTS antenna.
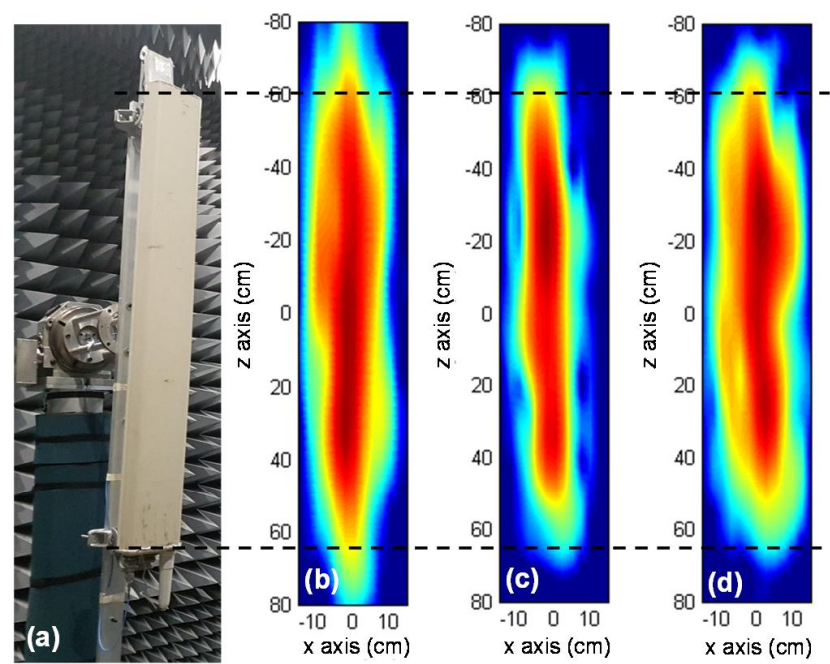

Malfunctioning BTS antenna.
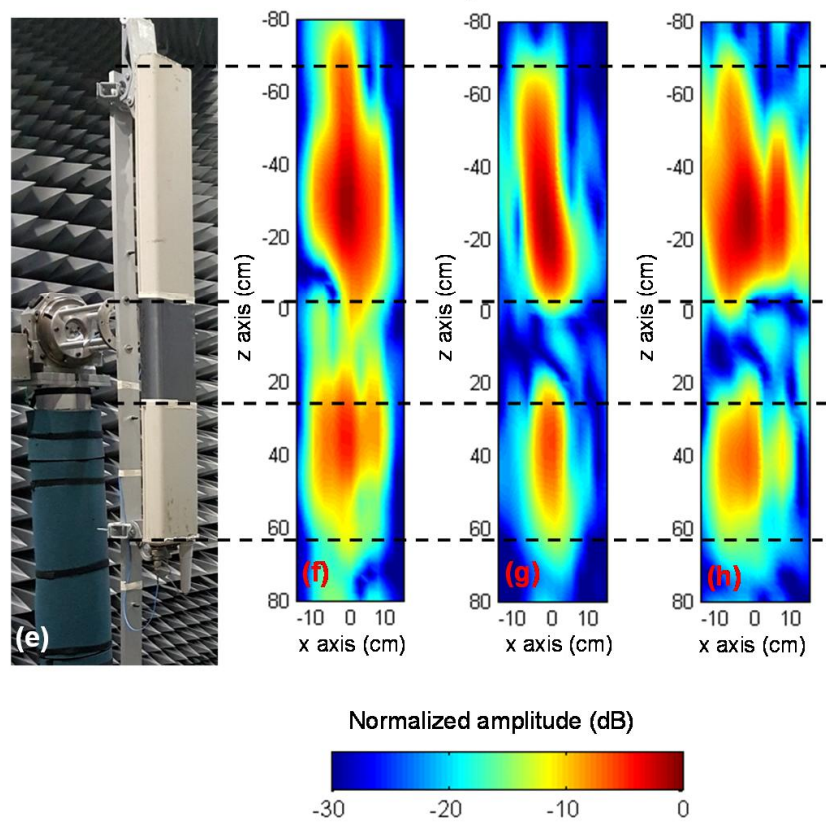

Fig. 4. Reconstructed equivalent currents on the BTS antenna aperture: $(b, f)$ from in-situ amplitude-only NF measurements; $(\mathrm{c}, \mathrm{g})$ from amplitude and phase NF measurements at spherical range in anechoic chamber; and $(\mathrm{d}, \mathrm{h})$ from amplitude-only NF measurements at spherical range in anechoic chamber. A picture of the BTS antenna is displayed on the left side of the figure without (a) and with (e) aluminum foil.

To illustrate this, the field radiated by the BTS antenna in a $100 \mathrm{~m} \times 100 \mathrm{~m}$ area in front of the antenna has been calculated from the reconstructed equivalent currents using in-situ measurements. Calculation is done with a full wave-based electromagnetic simulation software that takes the equivalent currents as an input. As the BTS antenna is completely characterized by its equivalent currents model, such model can be placed at a different height or orientation, e.g. $20 \mathrm{~m}$ above ground.
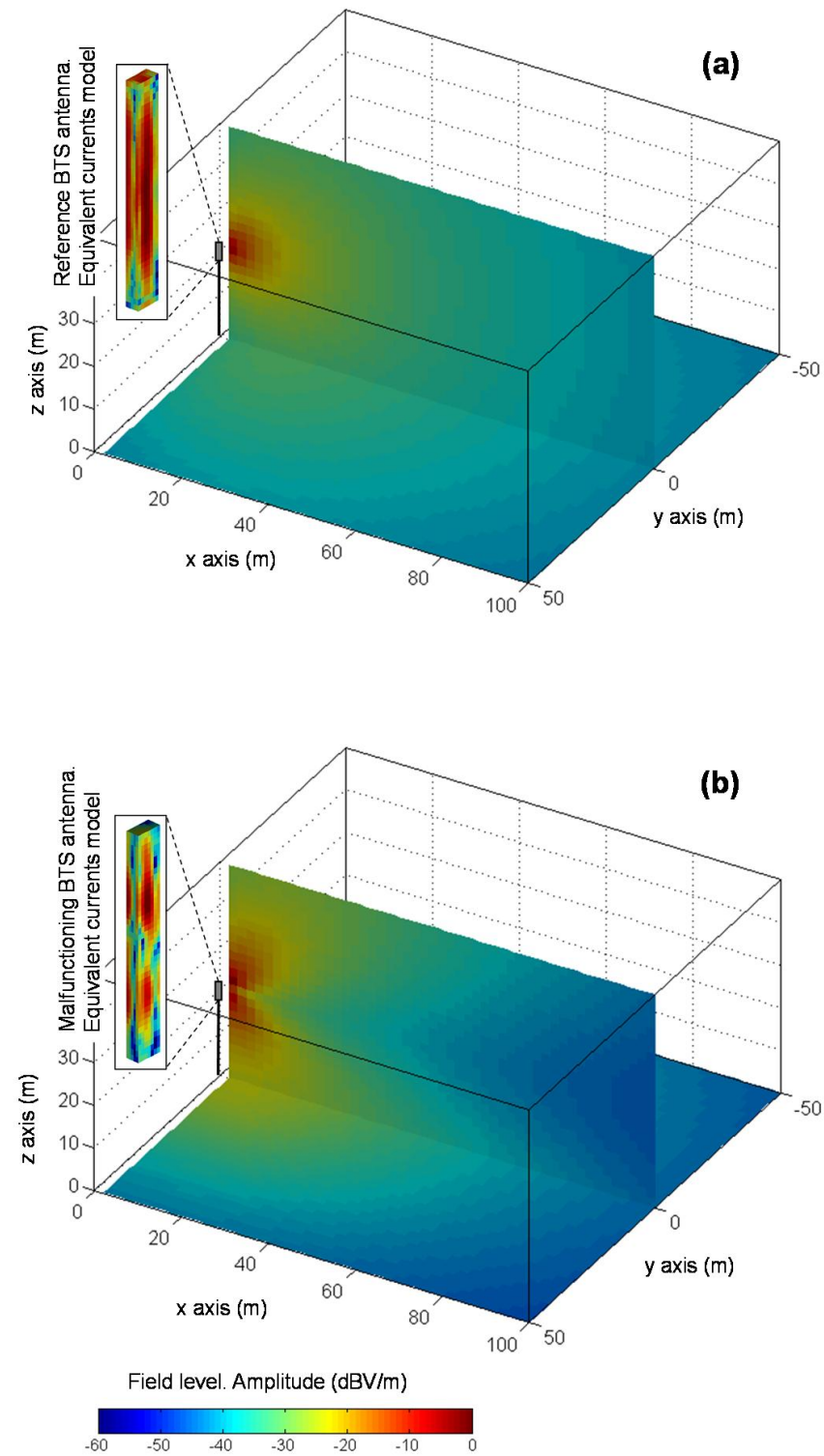

Fig. 5. Radioelectric coverage calculated from the reconstructed fields on the BTS antenna aperture. BTS antenna placed at height of $20 \mathrm{~m}$. (a) Reference BTS antenna. (b) Malfunctioning BTS antenna.

Predicted electric field levels are depicted in Fig. 5(a) for the BTS antenna in normal operation conditions, and in Fig. 5(b) for the malfunctioning BTS antenna. It can be noticed that the malfunctioning BTS antenna results in lower field levels than the reference antenna, due to the fact that its radiation pattern is less directive. Besides, the blockage due to the aluminum foil can still be noticed when evaluating the radiated field in a vertical plane in front of the BTS antenna aperture (Fig. 5(b)). From these results, mobile network operator can decide if the 
malfunctioning BTS antenna still provides enough coverage or if the radiated field levels are too low so it is better to replace the BTS antenna.

Concerning the accuracy of the proposed methodology, the Root Mean Square Error (RMSE) between the coverage level calculated from anechoic chamber measurements and from insitu UASAM measurements is $0.2 \%$.

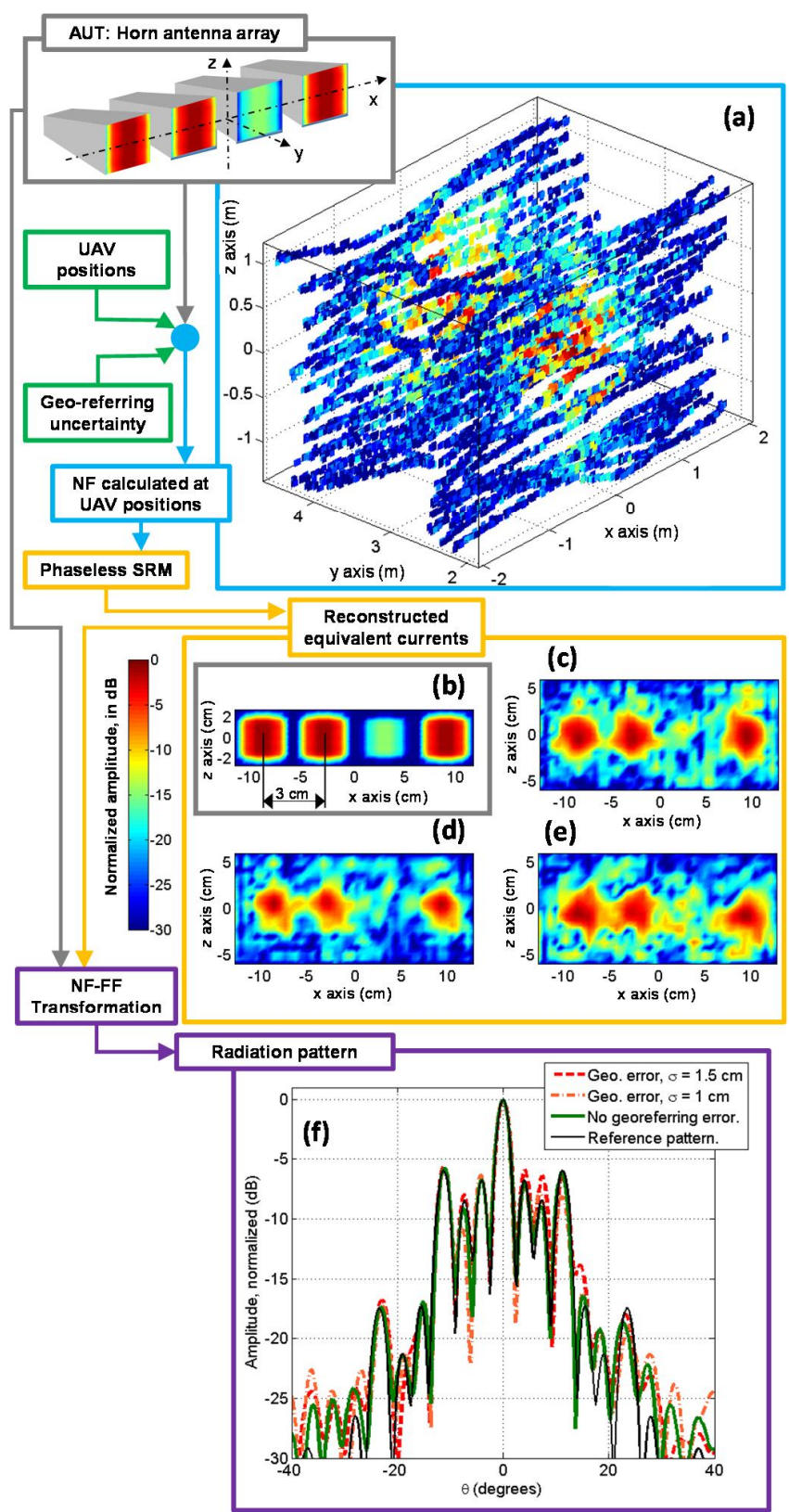

Fig. 6. Flowchart and results of antenna diagnostics and characterization at 25 GHz. (a) NF calculated at UAV positions. Reconstructed equivalent currents: (b) from NF measurements at anechoic chamber, (c) from NF calculated at UAV positions, without geo-referring error, (d) from NF calculated at UAV positions, with $\sigma=10 \mathrm{~mm}$ geo-referring error, (e) from NF calculated at UAV positions, with $\sigma=15 \mathrm{~mm}$ geo-referring error. (f) Calculated FF pattern, cut $\varphi$ $=0^{\circ}(\mathrm{XY}$ plane $)$.

\section{EVAluation Of THE SyStem PERFormance AT MILLIMETER-WAVE FREQUENCY BANDS}

In order to evaluate UASAM capabilities for antenna diagnostics and characterization at $5 \mathrm{G} \mathrm{mm}$-wave frequency bands, an application example consisting of a 4-element antenna array with non-uniform excitations working at $25 \mathrm{GHz}$ has been selected. The elements of the array are horn antennas, with a $3-\mathrm{cm}$ space between each of them

For the sake of clarity, the methodology followed in this application example is summarized in the flowchart shown in Fig. 6.

As the upper frequency limit of the power sensor currently onboard the UAV is $8 \mathrm{GHz}$, in this example the NF radiated by the AUT at the acquisition points had to be calculated from an equivalent magnetic currents model of the AUT measured at anechoic chamber (Fig. 6(b)), adding noise according to a Signal-to-Noise ratio of $30 \mathrm{~dB}$ (a level worse than the noise response of power detectors working up to $30 \mathrm{GHz}$ ).

Two planar measurement domains of $4 \mathrm{~m} \times 2 \mathrm{~m}$, placed at 2.5 and $4 \mathrm{~m}$ in front of the AUT aperture have been considered. Next, UAV flight path is defined, resulting in 7108 acquisition points on each domain. Once the UAV flight path is created, the UAV is taken off and set up to autonomous flight mode, recording the coordinates of the acquisition points. Then, the $\mathrm{NF}$ is calculated (using the AUT model) at the geo-referred positions, as depicted in Fig. 6(a).

Next, equivalent magnetic currents on the AUT aperture plane are recovered. When comparing the reference currents (Fig. 6(b)) with the reconstructed ones (Fig. 6(c)) it can be observed that the amplitude distribution of the array elements is recovered.

The impact of geo-referring errors has been assessed, modeling them as a normal probability distribution (being $\sigma$ is standard deviation). $\sigma=1 \mathrm{~cm}$ and $\sigma=1.5 \mathrm{~cm}$ geo-referring errors, which are within the degree of geo-referring uncertainty of the RTK system [11], have been introduced in the $\mathrm{x}, \mathrm{y}$, and $\mathrm{z}$ coordinates of the acquisition points. Results are depicted in Fig. 6(d) and Fig 6(e) respectively, where the antenna array elements can be still detected.

Finally, from the reconstructed equivalent currents, the AUT radiation pattern can be retrieved. Radiation pattern calculated from the equivalent currents depicted in Fig. 6(b)-(e) is plotted in Fig. 6(f). Even for a geo-referring error of $\sigma=1.5 \mathrm{~cm}$, discrepancies between the calculated radiation pattern and the reference one are less than $3 \mathrm{~dB}$.

\section{CONCLUSION}

Results presented in this contribution has proven the viability of using compact low-cost UAV-based antenna measurement systems for in-situ diagnostics and characterization of mobile network antennas. Quick deployment and ease-of-operation are two additional advantages of the proposed methodology.

The capability of conducting in-situ measurements for rapid assessment of the status of mobile networks commercial antennas will become a helpful tool for mobile network infrastructure maintenance tasks, especially in the case of denser networks as expected with $5 \mathrm{G}$. Here the disruptive 
improvement is given by measurements post-processing, that allows not only the detection of malfunctioning elements in mobile network antennas, but also the evaluation of the radioelectric coverage, enabling predictive maintenance of the mobile network infrastructure.

UASAM geo-referring accuracy is currently limited to 1-2 $\mathrm{cm}$, which has been shown to be sufficient for antenna diagnostics and characterization up to $25 \mathrm{GHz}$. In any case, sub$\mathrm{cm}$ geo-referring can be achieved by complementing current UASAM navigation system with an accurate geo-referring system (e.g. a laser tracker).

\section{ACKNOWLEDGEMENT}

This work has been developed under the framework of the Universidad de Oviedo degree Expert in Remotely Piloted and Autonomous Flight Aircrafts (www.dronesuniovi.es).

\section{REFERENCES}

[1] P. Demestichas, A. Georgakopoulos, D. Karvounas, K. Tsagkaris, V. Stavroulaki, J. Lu, C. Xiong, J. Yao, "5G on the horizon: key challenges for the radio-access network," IEEE Vehicular Technology Magazine, Vol. 8, No. 3, pp. 47-53, 2013.

[2] P. K. Agyapong, P. K., M. Iwamura, D. Staehle, W. Kiess, A. Benjebbour, "Design considerations for a $5 \mathrm{G}$ network architecture," IEEE Communications Magazine, Vol. 52, No. 11, pp. 65-75, 2014.

[3] Ulrich Rehfuess. "5G for people and things. $700 \mathrm{MHz}$ band as key to success for wide-area $5 \mathrm{G}$ services," ITU-Nokia report. Available online: https://www.itu.int/en/ITU-D/Regional-

Presence/Europe/Documents/Events/2017/Spectrum\%20Management/ Ulrich_Nokia_5G_in\%20700w.pdf [Accessed on October 9, 2017].

[4] N. Bhushan, J. Li, D. Malladi, R. Gilmore, D. Brenner, A. Damnjanovic, R. T. Sukhavasi, C. Patel, S. Geirhofer, "Network densification: the dominant theme for wireless evolution into $5 \mathrm{G}$," IEEE Communications Magazine, Vol. 52, No. 2, pp. 82-89, 2014.

[5] A. Sulyman, A. Nassar, M. Samimi, G. Maccartney, T. S. Rappaport, A. Alsanie, "Radio propagation path loss models for $5 \mathrm{G}$ cellular networks in the $28 \mathrm{GHz}$ and $38 \mathrm{GHz}$ millimeter-wave bands," IEEE Communications Magazine, Vol. 52, No. 9, pp. 78-86, 2014.

[6] J. A. Quijano, L. Scialacqua, J. Zackrisson, L. Foged, M. Sabbadini, G. Vecchi, "Suppression of Undesired Radiated Fields Based on Equivalent Currents Reconstruction From Measured Data," IEEE Antennas and Wireless Propagation Letters, Vol. 10, pp. 314-317, 2011.

[7] Y. Alvarez, F. Las-Heras, M. R. Pino, "The sources reconstruction method for amplitude-only field measurements," IEEE Transactions on Antennas and Propagation, Vol. 58, No. 8, pp. 2776-2781, 2010.

[8] S. Blanch, J. Romeu, A. Cardama, "Near field in the vicinity of wireless base-station antennas: an exposure compliance approach," IEEE Transactions on Antennas and Propagation, Vol. 50, No. 5, pp. 685-692, 2002.

[9] M. Mozaffari, W. Saad, M. Bennis, and M. Debbah, "Unmanned aerial vehicle with underlaid device-to-device communications: Performance and tradeoffs," IEEE Transactions on Wireless Communications, vol. 15 no. 6, pp. 3949-3963, June 2016

[10] M. Mozaffari, W. Saad, M. Bennis, and M. Debbah, "Efficient Deployment of Multiple Unmanned Aerial Vehicles for Optimal Wireless Coverage," IEEE Communications Letters, vol. 20, no. 8, pp. 1647-1650, August 2016.

[11] M. G. Fernández, Y. A. López, A. Arboleya, B. González-Valdés, Y. R. Vaqueiro, M. E. de Cos Gómez, F. Las-Heras, "Antenna Diagnostics and Characterization using Unmanned Aerial Vehicles," IEEE Access, 2017.

[12] G. Virone, A. M. Lingua, M. Piras, A. Cina, F. Perini, J. Monari, F. Paonessa, O. A. Peverini, G. Addamo, R. Tascone, "Antenna pattern verification system based on a micro unmanned aerial vehicle (UAV)", IEEE Antennas and Wireless Propagation Letters, Vol, 13, pp. 169-172, 2014.

[13] T. Fritzel, R. Strauss, H.-J. Steiner, C. Eisner, T. Eibert, "Introduction into an UAV-based Near-Field system for in-situ and large-scale antenna measurements," In IEEE Conference on Antenna Measurements and Applications (CAMA), October 23-27, 2016, Syracuse, USA. pp. 1-3.
[14] Microwave Vision Group (MVG). StarProd LTB (Defect Detection Bench). Product brochure. Available online: http://www.mvgworld.com/es/system/files/mvg_a4_brochure_starprodltb_en4_final_0. pdf [Accessed on October 10, 2017].

[15] A. Bisognin, N. Nachabe, C. Luxey, F. Gianesello, D. Gloria, J. Costa, C. Fernandes, Y. Álvarez, A. Arboleya, J. Laviada, F. Las-Heras, nDolatsha, B. Grave, M. Sawaby, A. Arbabian, "Ball Grid Array Module with Integrated Shaped Lens for $5 \mathrm{G}$ Backhaul/Fronthaul Communications in F-band," IEEE Transactions on Antennas and Propagation, Special issue on antennas and propagation aspects of 5G communications, Vol. 65, No. 12, pp. 6380-6394, December 2017

\section{BIOGRAPHIES}

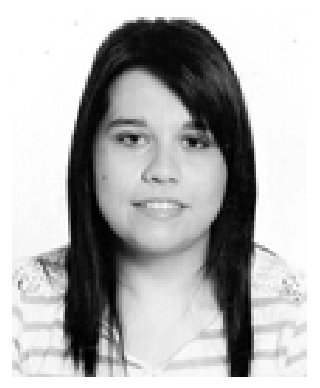

María García-Fernández (S'15) received the M.Sc. degree in telecommunication engineering from the University of Oviedo, Gijón, Spain, in 2016, where she is currently pursuing the Ph.D. degree. She was a Visiting Student with Stanford University (CA, USA), in 2013 and 2014. Since 2013, she has been involved in several research projects within the TSC-UNIOVI research group, University of Oviedo. Her current research interests include inverse scattering, remote sensing, radar, and imaging techniques.

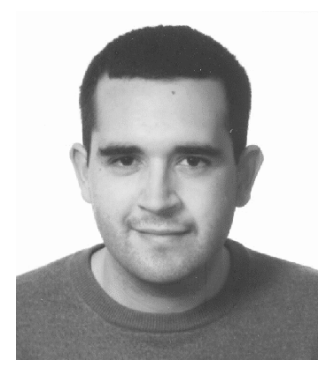

Yuri Alvarez (S'06 - M'09 - SM'15) received the M.S. and Ph.D. degrees in telecommunication engineering from the University of Oviedo, Gijón, Spain, in 2006 and 2009, respectively. He is currently an Assistant Professor with the TSC-UNIOVI research group, University of Oviedo, Gijón, Spain. His research interests include antenna diagnostics and measurement techniques, inverse scattering and imaging, and phaseless methods. Dr. Alvarez received 2011 Regional and National Awards to the Best Ph.D. Thesis on Telecommunication Engineering.

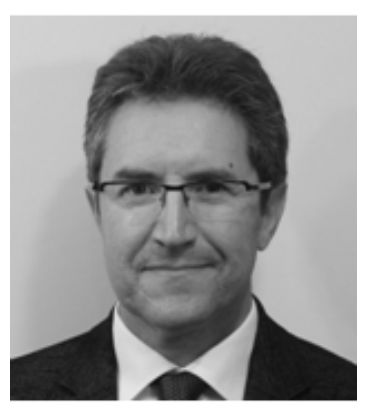

Fernando Las-Heras (M'86 SM'08) received the M.S. in 1987 and the Ph.D. in 1990, both in Telecommunication Engineering, from the Technical University of Madrid (UPM). From December 2003 he holds a Full-Professor position at the University of Oviedo where he was the Vice-dean for Telecommunication Engineering (2004-2008). As of 2001 he heads the TSC-UNIOVI research group at the University of Oviedo. He has authored over 300 articles published in academic journals and proceedings of international conferences. 